\title{
A New Parallel Quantum Genetic Algorithm with Probability-Gate and Its Probability Analysis
}

\author{
Shuxia $\mathrm{Ma}^{1}$ Weidong $\mathrm{Jin}^{2}$ \\ ${ }^{1}$ Department of Mathematics, Southwest Jiaotong University, Chengdu 610031, China \\ ${ }^{2}$ School of Electrical Engineering, Southwest Jiaotong University, Chengdu 610031, China
}

\begin{abstract}
In this paper, a novel Quantum Genetic Algorithm (QGA) called Parallel Probability-gate Quantum Genetic Algorithm (PPQGA) is proposed. The main points of PPQGA are that a new chromosome representation called qubit representation, a new updating gate called probability-gate and a novel evolutionary strategy being used in update procedure are introduced. Based on the concepts and principles of quantum computing introduced, PPQGA is characterized by rapid convergence, good global search capability and the ability of possessing exploitation simultaneously. The experimental results of several special functions show that PPQGA can speedup the migration of the top individuals of subpopulations and is superior to other several genetic algorithms greatly in quality and efficiency.
\end{abstract}

Keywords: Quantum genetic algorithm(QGA), Parallel probability-gate quantum genetic algorithm (PPQGA), Probability analysis

\section{Introduction}

Genetic Algorithm (GA) advanced by Prof. Holland in Michigan University in 1975 is called Basic Genetic Algorithm.(BGA). It is a powerful search technique that has been shown to robust optimization algorithms and has been used successfully to find adequate solutions to complex problems in numerous domains of science and engineering. However, there exist some problems in the utilization of GA that they can be very demanding in terms of computation and memory, and sequential GA may get trapped in a suboptimal region of the search space thus becoming unable to find better quality solutions. So Parallel Genetic Algorithms (PGAs) are proposed to solve more difficult problems which need large population [1]-[3].

Quantum computation is a newly emerging interdisciplinary science of information science and quantum science. In 1994, Shor proposed the first quantum algorithm to solve the difficult classic computing problem of prime factorization of large numbers. This algorithm can also be applied to RSA encryption technique [4]. In 1996, Grover proposed a quantum algorithm of random search in database, which can accelerate the search in untidy database by $\sqrt{N}$ order on the quantum computer [5]. Because of its unique characteristics of computation, quantum computation has attracted wide attention and soon become the research focus.

Quantum Genetic Algorithm (QGA) is the combination of quantum computation and genetic algorithm. At present, the research in this field concentrates mainly on two models: one is the Quantum Inspired Genetic Algorithm(QIGA) based on the quantum's multi-universe character [6], the other is the Genetic Quantum Algorithm (GQA), based on the superposition character of qubit and quantum state [7]. This paper proposes a Parallel Probability-gate Quantum Genetic Algorithm (PPQGA).

In the algorithm, the individuals in the universe are expressed by multi-state gene qubit encoding method. In the individual's updating, the general quantum rotation gate strategy is replaced by the probability-gate strategy, in which every updating is made certain by a probability matrix instead of the general quantum rotation gate strategy. The rationality of the probability matrix is analyzed in the paper and the experimental results of several special functions are listed to indicate that PPQGA is superior to other several genetic algorithms greatly in quality and efficiency.

\section{Quantum genetic algorithm}

The QGA is based on the representation of the quantum vector. It applies the probabilistic amplitude representation of qubit to the coding of chromosome so that one chromosome can represent the superposition of many states. With the update operation of chromosome by quantum rotation gate, it eventually reaches the optimum resolution of the aim.

\subsection{Qubit encode}


In quantum computer, the smallest information location is a two-state quantum system, called qubit. In QGA, qubit is used to store and represent one gene. This gene may be in the ' 1 ' state, the ' 0 ' state, or any superposition of the two. That is to say, the information represented by this gene is not stable, but probable. The characteristic of the representation is that any linear superposition of solutions can be represented. Therefore, when an operation is carried out on this gene, it may be done to all probable information simultaneously.

\subsection{The structure of QGA}

The smallest unit of information stored in two-state. The state of a qubit can be represented as $|\psi\rangle=$ $|\alpha\rangle+|\beta\rangle$ where $\alpha$ and $\beta$ are probability amplitudes of the corresponding states. Normalization of the state to unity guarantees $|\alpha|^{2}+|\beta|^{2}=1$, where $|\alpha|^{2}$ gives probability that qubit will be found in ' 0 'state and $|\beta|^{2}$ gives probability that qubit will be found in ' 1 'state. According to this, a system with $m$ qubits can contain information of $2^{m}$ states and any linear superposition of all possible states can be represented as follows:

$$
\left[\begin{array}{c|c|c|c}
\alpha_{1} & \alpha_{2} & \cdots & \alpha_{m} \\
\beta_{1} & \beta_{2} & \cdots & \beta_{m}
\end{array}\right] \quad\left|\alpha_{\mathrm{i}}\right|^{2}+\left|\beta_{\mathrm{i}}\right|^{2}=1(i=1,2, \ldots, m)
$$

The basic structure of QGA is described as follows:

1. Initialize colony $Q(t)$, and $t=0$;

2. Create $P(t)$ by measuring $Q(t)$ states;

3. evaluate $P(t)$;

4. Store the best individual among $P(t)$ and its fitness;

5. Stop condition: The condition is satisfied then output the best one, if not, go on;

6. Update $Q(t)$ with quantum rotation gate to get son colony $Q(\mathrm{t}+1)$, then $t=t+1$, go to 2 .

The $Q(t)$ is the $t$-th generation colony of quantum chromosomes, $P(t)$ is the linear superposition of all possible states of $t$-th generation colony. When the colony is initialized, all the quantum chromosomes have the same value $1 / \sqrt{2}$, it means that a quantum chromosome represents all possible superposition states with the same probability. In step 2, there is a random number belong to [0,1], if it bigger than $\left|\alpha_{i}^{t}\right|^{2}$, then it is endowed with 1 , or endowed with 0 . In step 6 , updating $Q(t)$ with appropriate quantum gate to make the quantum chromosomes have better state.

\subsection{Qubit rotation gate updating strategy}

As the updating execution mechanism, the quantum gate can be designed in compliance with the practical problem. Though many quantum gates can be selected, quantum rotation gate is preferable due to the calculation character of QGA.

Commonly, $Q(t)$ is updated with quantum rotation gate which is chosen as quantum logic gate through the formula as:

$$
\left[\begin{array}{c}
\alpha_{i}^{t+1} \\
\beta_{i}^{t+1}
\end{array}\right]=\left[\begin{array}{cc}
\cos \left(\Delta \theta_{i}\right) & -\sin \left(\Delta \theta_{i}\right) \\
\sin \left(\Delta \theta_{i}\right) & \cos \left(\Delta \theta_{i}\right)
\end{array}\right]\left[\begin{array}{c}
\alpha_{i}^{t} \\
\beta_{i}^{t}
\end{array}\right]
$$

where $\Delta \theta$ is the rotation angle of quantum rotation gate and often be get from lookup table of QGA for convergence.

\begin{tabular}{|cc|c|c|c|c|c|c|}
\hline & & \multirow{2}{*}{$x_{i} b_{i}$} & & & \multicolumn{4}{|c|}{$s\left(\alpha_{i} \beta_{i}\right)$} \\
\cline { 4 - 7 } & & & & & \\
& & $\Delta(b)$ & $\theta_{i}$ & $\alpha_{i} \beta_{i}$ & $\alpha_{i} \beta_{i}$ & $\alpha_{i}$ & $\beta_{i}$ \\
$<0$ & $<0$ & $=0$ & $=0$ \\
& & & & & & \\
\hline & & & & & & & \\
0 & 0 & false & 0 & 0 & 0 & 0 & 0 \\
0 & 0 & true & 0 & 0 & 0 & 0 & 0 \\
0 & 1 & false & 0 & 0 & 0 & 0 & 0 \\
0 & 1 & true & $0.05 \pi$ & -1 & +1 & \pm 1 & 0 \\
0 & 0 & false & $0.05 \pi$ & -1 & +1 & \pm 1 & 0 \\
0 & 0 & true & $0.05 \pi$ & +1 & -1 & 0 & \pm 1 \\
1 & 1 & false & $0.05 \pi$ & +1 & -1 & 0 & \pm 1 \\
1 & 1 & true & $0.05 \pi$ & +1 & -1 & 0 & \pm 1 \\
& & & & & & & \\
\hline
\end{tabular}

Table 1: Lookup table of QGA.

where $x_{i}$ and best $_{i}$ is the $i$-th bit of $x$ and best individual separately, $f(x)$ is fitness of $x, s\left(\alpha_{i} \beta_{i}\right)$ is sign of $\theta_{i}$.

\section{Parallel probability-gate quantum genetic algorithm}

This paper proposes a Parallel Probability-gate Quantum Genetic Algorithm that adopts a multiuniverse parallel Structure [8]. In this structure, different universes make the evolutionary towards their own different aims, and the excellent individuals in different circumstance among the universes exchange through emigration and quantum crossover mechanism. And a novel evolutionary strategy with probability-gate is used in update procedure. All these will effectively overcome the premature convergence, and have higher convergence speed and precision than QGA. 


\subsection{Probability-gate and its structure}

From the structure of QGA know that QGA evolves quantum chromosomes by some quantum gates and creates binary system values by measuring $\left|\alpha_{i}^{t}\right|^{2}$. This paper proposes probability-gate which update $\left[\begin{array}{l}\left|\alpha_{i}^{t}\right|^{2} \\ \left|\beta_{i}^{t}\right|^{2}\end{array}\right]$ to $\left[\begin{array}{l}\left|\alpha_{i}^{t+1}\right|^{2} \\ \left|\beta_{i}^{t+1}\right|^{2}\end{array}\right] \quad i=1,2, \cdots, m$ by probability matrix, by which the processing time of search satisfactory solutions of optimization problems is greatly reduced. Furthermore, the quantum gate is random, and to a great extent, the individual could degenerate or the colony gets trapped in a suboptimal region of the search space thus becoming unable to find better quality solutions. By probability-gate, the former information of the best individual to overlap to quantum chromosomes will be reserved for making the $\mathrm{P}(\mathrm{t})$ has better filial generation.

Definition 1 If quantum chromosomes $Q(t)$ are

updated by $\left[\begin{array}{l}\left|\alpha_{i}^{t+1}\right|^{2} \\ \left|\beta_{i}^{t+1}\right|^{2}\end{array}\right]=\mathrm{A}\left[\begin{array}{l}\left|\alpha_{i}^{t}\right|^{2} \\ \left|\beta_{i}^{t}\right|^{2}\end{array}\right]$

then $A$ is called a probability-gate.

In this definition, the $i$-th qubit of the $t+1$-th generation individual is obtained by multipling probability matrix A and the $i$-th qubit of the $t$-th generation individual straitly. It effectively avoids continually refering lookup table. Next theorem describes the structure of (3) is reasonable.

If define $\frac{1}{\mu(i)}$ as adjusting quantity of the $i$-th qubit, commonly its value depends on the situation of the $i$-th bit. $f(b)$ is the best individual of last generation. $x_{i}$ and $b_{i}$ is the $i$-th bit of the best individual in this generation and in last generation separately. The probability-gate is created as follows:

When $f(x) \geq f(b)$, or $f(x)<f(b)$ with $x_{i}=b_{i}$, then $\quad \mathrm{A}=\left[\begin{array}{ll}1-a & a \\ a & 1-a\end{array}\right]$

When $f(x)<f(b)$ with $x_{i} \neq b_{i}$,

then

$$
\mathrm{A}=\left[\begin{array}{ll}
a & 1-a \\
1-a & a
\end{array}\right]
$$

while $\quad a=\frac{(-1)^{b_{i}}}{2 \mu(i)\left(1-2\left|\alpha_{i}^{t}\right|^{2}\right)}$

According this structure, $Q(t)$ is updated by judgement of $f(x), f(b), x$ and $b$ in spite of refering lookup table which is lack of pertinence. Noticing formual (6) and $\left|\beta_{i}^{t}\right|^{2}=1-\left|\alpha_{i}^{t}\right|^{2}$, the theorem will be easily gained as follows:

Theorem 1 If $Q(t)$ is updated according with above probability-gate $A$, then:

When $f(x) \geq f(b)$, then

$$
\left|\alpha_{i}^{t+1}\right|^{2}= \begin{cases}\left|\alpha_{i}^{t}\right|^{2}+\frac{1}{2 \mu(i)} & b_{i}=0 \\ \left|\alpha_{i}^{t}\right|^{2}-\frac{1}{2 \mu(i)} & b_{i}=1\end{cases}
$$

When $f(x)<f(b)$, then

$$
\left|\alpha_{i}^{t+1}\right|^{2}= \begin{cases}\left|\alpha_{i}^{t}\right|^{2}+\frac{1}{2 \mu(i)} & b_{i}=x_{i}=0 \\ \left|\alpha_{i}^{t}\right|^{2}-\frac{1}{2 \mu(i)} & b_{i}=x_{i}=1 \\ \left|\beta_{i}^{t}\right|^{2}+\frac{1}{2 \mu(i)} & b_{i} \neq x_{i}=1 \\ \left|\beta_{i}^{t}\right|^{2}-\frac{1}{2 \mu(i)} & b_{i} \neq x_{i}=0\end{cases}
$$

At the same time, $\quad\left|\beta_{i}^{t+1}\right|^{2}=1-\left|\alpha_{i}^{t+1}\right|^{2}$.

Proof. To prove theorem, when $f(x) \geq f(b)$, we note that formula (3) is expressed as

$$
\left[\begin{array}{l}
\left|\alpha_{i}^{t+1}\right|^{2} \\
\left|\beta_{i}^{t+1}\right|^{2}
\end{array}\right]=\left[\begin{array}{ll}
1-a & a \\
a & 1-a
\end{array}\right]\left[\begin{array}{l}
\left|\alpha_{i}^{t}\right|^{2} \\
\left|\beta_{i}^{t}\right|^{2}
\end{array}\right] .
$$

It implise that

$$
\begin{aligned}
\left|\alpha_{i}^{t+1}\right|^{2} & =(1-a)\left|\alpha_{i}^{t}\right|^{2}+a\left|\beta_{i}^{t}\right|^{2} \\
& =(1-a)\left|\alpha_{i}^{t}\right|^{2}+a\left(1-\left|\alpha_{i}^{t}\right|^{2}\right) . \\
& =\left|\alpha_{i}^{t}\right|^{2}-2 a\left|\alpha_{i}^{t}\right|^{2}+a
\end{aligned}
$$

Follows from (4) and note that formual (6), then we get 


$$
\begin{aligned}
\left|\alpha_{i}^{t+1}\right|^{2} & =\left|\alpha_{i}^{t}\right|^{2}+\left(1-2\left|\alpha_{i}^{t}\right|^{2}\right) \frac{(-1)^{b_{i}}}{2 \mu(i)\left(1-2\left|\alpha_{i}^{t}\right|^{2}\right)} \\
& =\left|\alpha_{i}^{t}\right|^{2}+\frac{(-1)}{2 \mu(i)}
\end{aligned}
$$

Namely, the first part of the theorem is gained.

As well as

$$
\left|\beta_{i}^{t+1}\right|^{2}=1-\left|\alpha_{i}^{t+1}\right|^{2}
$$

and the rest of the theorem is gained with the same way. The theorem shows that the construct of matrix A, just as formula (4), is reasonable.

The theorem denotes that when $X$ is not superior to $b$ we increase the probability of $\mathrm{Q}(\mathrm{t})$ according $b$ to embody character of $b$ whereas embody character of $x$.

\subsection{Probability analysis}

If the individuals of $P(t)$ is $m$ bits, for its former bits decides the individual be or not be in suboptimal region, and for every bit getting value 0 or 1 from $\left|\alpha_{i}^{t}\right|^{2}$ is subject to uniformity distributing in $[0,1]$, when $Q(t)$ creates $P(t)$, the difference probability of the $t$-th bit of individual with $b$ is:

$$
\begin{aligned}
& p_{i}=\min \left\{\left|\alpha_{i}^{t}\right|^{2},\left|\beta_{i}^{t}\right|^{2}\right\}=b_{i}\left|\alpha_{i}^{t}\right|^{2}+\left(1-b_{i}\right)\left(1-\left|\alpha_{i}^{t}\right|^{2}\right) \\
& (i=1,2, \cdots, m)
\end{aligned}
$$

The homology probability of the $t$-th bit of individual with $b$ is:

$$
\begin{aligned}
\overline{p_{i}} & =\max \left\{\left|\alpha_{i}^{t}\right|^{2},\left|\beta_{i}^{t}\right|^{2}\right\} \\
& =\left(1-b_{i}\right)\left|\alpha_{i}^{t}\right|^{2}+b_{i}\left(1-\left|\alpha_{i}^{t}\right|^{2}\right)
\end{aligned}
$$

The probability of the individual just being $b$ is:

$$
\begin{aligned}
p_{b} & =\prod_{i=1}^{m} \max \left\{\left|\alpha_{i}^{t}\right|^{2},\left|\beta_{i}^{t}\right|^{2}\right\} \\
& =\prod_{i=1}^{m}\left[\left(1-b_{i}\right)\left|\alpha_{i}^{t}\right|^{2}+b_{i}\left(1-\left|\alpha_{i}^{t}\right|^{2}\right)\right]
\end{aligned}
$$

For many local regions problem, when the radius of the smallest local region is $2^{(m-k)+1}-1$, the probability of the individual must being in local region is: $\bar{p}=\prod_{i=1, \cdots, k}\left(1-p_{i}\right)$. If $b$ already is best point in whole region, then $p_{i}=\min \left(\left|\alpha_{i}^{t}\right|^{2},\left|\beta_{i}^{t}\right|^{2}\right) \rightarrow 0$ while $t \rightarrow \infty$. That is $\bar{p} \rightarrow 1$. It shows this region is efficient. When the radius of the biggest local region is $2^{(m-l)+1}-1$, then the probability of the individual must getting out the local region is: $p=1-\prod_{i=1, \cdots, l}\left(1-p_{i}\right)$. So when $p_{i} \rightarrow 0 \quad(\forall i)$, $p \rightarrow 0$.

From all above analysis, the update matrix $A$ could make ensure that the next generation will includes $b$ in it or get out the local region and finds better one. Both of these conclusions are we wanted.

Now the basic structure of PPQGA is described as follows:

1. Initialize colony $Q(t)$, and $t=0$;

2. Initialize the genus $Q(t)$ and create $P(t)$ by $Q(t)$;

3. Save the best value of $P(t)$;

4. $t \leftarrow t+1$;

5. Create $P(t)$ by $Q(t-1)$;

6. Judge of $P(t)$ then get the best individual $x$;

7. Compare $x$ with $b$ and get quantum probabilitygate $A$, save the best individual in $b$;

8. Stop condition: the condition is satisfied, then output the best one, if not, go on; Renovate $Q(t)$ by $A$, $t=t+1$, then go to 4 .

\section{Model test functions}

(1) De Jong function:

$$
\begin{aligned}
& F_{1}=100\left(x_{1}^{2}-x_{2}\right)^{2}+\left(1-x_{1}\right)^{2} \\
& -2.048 \leq x_{i} \leq 2.048, i=1,2
\end{aligned}
$$

It is a 2-dim function. It has only one minimum value $f(1,1)=0$. Though this function is a singlepeak-value function, but it is morbidity function and it is difficult to optimize.

(2) Six-peaks-value-hunchback function:

$$
\begin{gathered}
F_{2}=\left(4-2.1 x_{1}^{2}+\frac{1}{3} x_{1}^{4}\right) x_{1}^{2}+x_{1} x_{2}+\left(-4+4 x_{2}^{2}\right) x_{2}^{2} \\
-3 \leq x_{1} \leq 3,-3 \leq x_{2} \leq 3
\end{gathered}
$$

This function has six local minimum values, and two of them are the global minimum value:

$$
\begin{aligned}
f & =(-0.0898,0.7126)=f(-0.0898,-0.7129) \\
& =-1.031628
\end{aligned}
$$

(3) Many-peaks-value function:

$$
\begin{aligned}
F_{3}= & x_{1}^{2}+2 x_{2}^{2}-10 \sin \left(2 x_{1}\right) \sin \left(x_{3}\right)+0.5 \cos \left(x_{1}+2 x_{2}\right) \\
& +x_{1}^{2} x_{3}^{2}-5 \sin \left(2 x_{1}-x_{2}+3 x_{3}\right) \\
& \quad-10 \leq x_{i} \leq 10 \quad \mathrm{i}=1,2,3
\end{aligned}
$$

This function has many local minimum values and one global minimum point $(-0.676256,-0.381582$, 1.282868), and global minimum value is 12.76547355.

With GA, QGA and PPQGA, we have experimented 50 times with stopping condition: Not 
stop until 100 generations in the experimentation. The scale of colony is 50 and $\mu(i)=\frac{1}{200-2 i}$. The result is shown as follows:

\begin{tabular}{|c|c|c|c|c|c|c|}
\hline & Algorithm & Precision & $\mathrm{TT}$ & $\mathrm{AT}$ & FT & FFT \\
\hline $\mathrm{F}_{1}$ & $\begin{array}{l}\text { GA } \\
\text { QGA } \\
\text { PPQGA }\end{array}$ & $1 \times 10^{-6}$ & 50 & 100 & $\begin{array}{l}11 \\
32 \\
43\end{array}$ & $\begin{array}{l}2 \\
21 \\
37\end{array}$ \\
\hline $\mathrm{F}_{2}$ & $\begin{array}{l}\text { GA } \\
\text { QGA } \\
\text { PPQGA }\end{array}$ & $1 \times 10^{-6}$ & 50 & 100 & $\begin{array}{l}27 \\
39\end{array}$ & $\begin{array}{l}2 \\
15 \\
29 \\
\end{array}$ \\
\hline $\mathrm{F}_{3}$ & $\begin{array}{l}\text { GA } \\
\text { QGA } \\
\text { PPQGA }\end{array}$ & $1 \times 10^{-6}$ & 50 & 100 & $\begin{array}{l}4 \\
21 \\
31 \\
\end{array}$ & $\begin{array}{l}1 \\
7 \\
19 \\
\end{array}$ \\
\hline
\end{tabular}

Table 2: The optimize results of these kinds of algorithm.

where

TT: The times of test

AT: The times of alternating

FT: The times of finding the optimize

FFT: Times for the optimum is found in first 50 generation

Precision: Times for the optimum is found

\section{Conclusions}

This paper proposes a new parallel evolutionary algorithm PPQGA with the qubit representation, the strategies of updating quantum gate using probabilitygate. The analysis of the structure with probability theory is given in paper. PPQGA is based on the principles of quantum computing such as concepts of qubits and superposition of states. It can represent a linear superposition of states. PPQGA is characterized by rapid convergence, good global search capability and short computing time. The experimental results of three special functions demonstrate that PPQGA is superior to GA, QGA in efficiency and quality.

\section{References}

[1] F. D. Toro and J. Ortega etc., PSFGA: a parallel genetic algorithm for multiobjective optimization, Proceedings of the $10^{\text {th }}$ EuromicroWorkshop on Parallel, Distributed and Networkbased Processing, pp. 384-391, 2002.

[2] M. AtiqullahM, Problem independent parallel genetic algorithm for design optimization, Proceedings of the international parallel and distributedp rocessing symposium, pp. 204-211, 2002.
[3] M. Nowostawski and R. Poli. Parallel Genetic Algorithm Taxonomy. 1999 Third International Conference on Knowledge-Based Intelligent Infonnation Engineering System, pp. 88-92, 1999.

[4] P.W. Shor, Algorithms for Quantum Computation:Discrete Logarithms and Factoring, Proceedings of the 35th Annual Symposium on the Foundation of Computer Sciences, pp. 20-22, 1994.

[5] L.K. Grover, A Fast Quantum Mechanical Algorithm for Database Search, Proceedings, 28th Annual ACM Symposium on the Theory of Computing, ACM Press, pp. 212-221, 1996.

[6] A. Narayanan and M. Moore, Quantum Inspired Genetic Algorithms, In Proceedings of the 1996 IEEE Intemational Conference on Evolutionary Computation, pp. 41-46, 1996.

[7] K.H. Han, Genetic Quantum Algorithm and Its Application to Combinatorial Optimization Problem, IEEE Proc. Of the 2000 Congress on Evolutionary Computation, pp. 1354-1360, 2000.

[8] J.A. Yang and B. Li, Multi-universe parallel quantum genetic algorithm and its application to blind source separation, IEEE Int. Conf. Neural Networks \&Signal Processing, pp. 393-398, 2003. 